THURSDAY, 14 JUNE 2018

\section{The EULAR Exercise recommendations for physical activity in people with inflammatory arthritis and osteoarthritis}

\section{SP0084 WHY PHYSICAL ACTIVITY EXERCISE WORKS}

M.D. Iversen. Department of Physical Therapy, Movement and Rehabilitation Sciences, Northeastern University, Bouve College of Health Sciences, Boston, USA

Pain, inflammation, and decreased physical function are consequences of musculoskeletal disorders (MSDs), limiting patients' daily activities and restricting their participation in society. Physical activity and exercise have positive health effects for the circulatory, digestive, endocrine, excretory, immune, integumentary, muscular, nervous, respiratory and skeletal systems. Given the benefits of physical activity and exercise, physical activity is a primary component of symptom management for individuals with musculoskeletal disorders. This presentation will review the short- and long-term effects of physical activity and exercise in general and specifically, for individuals with musculoskeletal disorders, with a focus on the cardiovascular, immune and musculoskeletal systems.

Disclosure of Interest: None declared

DOI: 10.1136/annrheumdis-2018-eular.7837

\section{SP0085 THE EVIDENCE FOR PHYSICAL ACTIVITY IN INFLAMMATORY ARTHRITIS AND OSTEOARTHRITIS}

A.-K. Rausch $^{1,2}$, on behalf of Task Force Members of the EULAR endorsed project "EULAR recommendations for physical activity in people with inflammatory arthritis and osteoarthritis". 'Department of Orthopaedics, Rehabilitation and Physical Therapy, Leiden University Medical Center, Leiden, Netherlands; ${ }^{2}$ Institut of Physiotherapy, Zurich University of Applied Sciences, Winterthur, Switzerland

Objective: To evaluate the effectiveness of physical activity in people with rheumatoid arthritis, spondyloarthritis and osteoarthritis.

Methods: A Systematic review and meta-analysis were performed searching he databases Pubmed/Medline, CENTRAL, Embase, Web of Science, Emcare, Psyclnfo up to April 2017. We included randomised controlled trials (RCT)s in adults $(\geq 18$ years) with rheumatoid arthritis $(R A)$, spondyloarthritis $(\mathrm{SpA})$ and osteoarthritis $(\mathrm{OA})$ investigating the effect of exercise or physical activity (PA) promotion on cardiovascular fitness, muscle strength, flexibility, neuromotor performance, and daily PA. Additionally we applied public health recommendations for PA based on the American College of Sports Medicine (ACSM) principles for effective interventions. Outcomes included assessments done directly after the intervention; If suitable, pooled in a meta-analysis using a random-effect model presented as standardised mean difference (SMD). Study registration in PROSPERO (CRD42017082131).

Results: The systematic review included $63 \mathrm{RCTs}$, of which 44 (3469 people with $\mathrm{RA}, \mathrm{SpA}, \mathrm{OA}$ ) were included in the meta-analysis. Moderate effects were found of aerobic exercises and resistance training on cardiovascular fitness (SMD 0.6 (95\% Cl 0.38 to 0.81$)$ ) and muscle strength (SMD 0.54 (95\% Cl 0.35 to 0.72$)$ ) respectively, but no effect of combined exercises on flexibility (SMD $0.12(95 \% \mathrm{Cl}$ -0.16 to 0.41$)$ ). PA promotion interventions produced a small increase in PA behaviour (SMD 0.21 (95\% $\mathrm{Cl} 0.03$ to 0.38$)$ ).

Conclusion: Exercising and PA promotion according to public recommendations for PA were effective on cardiovascular fitness and muscle strength, with moderate, and thus clinically relevant effect sizes in people with $\mathrm{SpA}, \mathrm{RA}$ and $\mathrm{OA}$.

Disclosure of Interest: None declared

DOI: 10.1136/annrheumdis-2018-eular.7804

\section{\begin{tabular}{|l|l}
\hline SP0086 THE DEVELOPMENT AND PURPOSE OF THE EULAR \\
\hline
\end{tabular} RECOMMENDATIONS FOR PHYSICAL ACTIVITY IN PEOPLE WITH INFLAMMATORY ARTHRITIS AND OSTEOARTHRITIS}

K. Niedermann, on behalf of Task Force of the project "EULAR recommendations for physical activity in people with inflammatory arthritis and osteoarthritis". School of Health Professions, Zurich University of Applied Sciences, Winterthur, Switzerland

Objective: Regular physical activity (PA) is increasingly promoted for people with rheumatic and musculoskeletal diseases as well as the general population. We evaluated if the public health recommendations for PA are applicable for people with inflammatory arthritis (iA; Rheumatoid Arthritis and Spondyloarthritis) and osteoarthritis (OA) in order to develop evidence-based recommendations for advice and guidance on PA in clinical practice.

Methods: The EULAR standardised operating procedures for the development of recommendations were followed. A task force (including rheumatologists, other medical specialists and physicians, health professionals, patients and methodologists) from 16 countries met twice. In the first task force meeting, 13 research questions to support a systematic literature review (SLR) were identified and defined. In the second meeting, the SLR evidence was presented and discussed before the recommendations, research agenda and education agenda were formulated.

Results: The task force developed and agreed on four overarching principles and ten recommendations for PA in people with IA and OA. The mean level of agreement between the task force members ranged between 9.8 to 8.8. Given the evidence for its effectiveness, feasibility and safety, PA is advocated as an integral part of standard care throughout the course of these diseases. Finally, the task force agreed on a related research and education agenda.

Conclusion: Evidence and expert opinion inform these recommendations to provide guidance in the development, conduct and evaluation of PA interventions and promotion in patients with $\mathrm{iA}$ and $\mathrm{OA}$. It is advised that these recommendations should be implemented considering individual needs and national health systems.

Disclosure of Interest: None declared

DOI: 10.1136/annrheumdis-2018-eular.7807

\section{SP0087 HOW TO DEAL WITH CARDIOVASCULAR RISK FACTORS FOR PHYSICAL ACTIVITY AND IMPLEMENT THE PA RECOMMENDATIONS IN THE RHEUMATOLOGIC PRACTICE?}

M. Nurmohamed, on behalf of E-RAC study Group: Edelaar L, van der Esch M, Nurmohamed M. Amsterdam Rheumatology immunology Center / VUmc and Reade, Amsterdam, Netherlands

How to deal with cardiovascular risk factors for physical activity and implement the PA recommendations in the rheumatologic practice?

Nowadays, physical activity is increasingly advocated not only for the general population but also for patients with inflammatory arthritis as well as osteoarthritis Generally, physical activity has favourable effects on cardiovascular risk factors. A recent systematic review revealed moderate, statistically significant improvements in blood pressure, LDL-cholesterol, and total cholesterol levels after about one year (additional) physical activity. Also the BMI improved and the effects appear to be a dose-related but not with all cardiovascular risk factors. Nevertheless, these favourable modest effects on cardiovascular risk factors could result in a clinically relevant reduction of cardiovascular events, provided that the physical activity remains enhanced albeit that thus far the evidence towards a reduction of cardiovascular events is not equivocal.

It is important to realise that this review generally comprised persons without cardiovascular disease or risk factors for cardiovascular disease. It's well known that (very) high intensity exercise results in a small absolute increase of myocardial infarction in comparison to those who have less strenuous physical exertion. The risk is the highest in those who exercised less than one time per week.

Therefore, the American College of Sports Medicine (ACSM) developed an exercise preparticipation health screening process to mitigate these risks. Recommendations were developed for those 1) who should receive medical permission before starting a physical exercise program 2) with clinically significant disease(s) who may benefit from physical activity under medical supervision and 3) with medical conditions that excludes them from participation in physical activity programs The health screening advices of the ASCM are based on an health screening logic model for aerobic exercise participation that takes the current level of physical activity, 2) presence of signs or symptoms and/or known cardiovascular disease/ risk factors and 3) desired physical activity into account.

However, the ASCM guidelines do not apply for patients with inflammatory joint disorders such as rheumatoid arthritis (RA). RA is associated with increased overall mortality compared to the general population, with cardiovascular diseases as one of the main causes.

The optimisation of management of cardiovascular risk in RA patients is an important aim in the treatment. Active counselling is indispensable, including also attention to exercise, particularly in RA patients with a high CV risk, defined as a 10 year CV risk of $20 \%$ or higher. Physical exercise for these patients is necessary and challenging since professionals should take multiple factors into account, such as comorbid conditions related to CV risk (e.g. hypertension, diabetes mellitus and obesity). However, the effects of exercise therapy on CV risk in RA patients are unknown and the required intensity is also unknown. 